\title{
Sobrevivência e reprodução de Synthesiomyia nudiseta (Diptera, Muscidae) ${ }^{1}$
}

\author{
Rodrigo F. Krüger ${ }^{2}$, Tiago K. Krolow ${ }^{3}$, Rodrigo R. Azevedo ${ }^{4}$, Juliano L. P. Duarte ${ }^{5} \&$ Paulo B. Ribeiro ${ }^{5}$ \\ 1. Contribuição $\mathrm{N}^{\circ} 1586$ do Departamento de Zoologia da Universidade Federal do Paraná (UFPR). \\ 2. Campus de Rio Paranaíba, Universidade Federal de Viçosa, Caixa Postal 22, 38810-000 Rio Paranaíba, MG, Brasil. (rfkruger@ufv.br) \\ 3. Departamento de Entomologia, Instituto Nacional de Pesquisas da Amazônia, Av. André Araújo, 2936, 69083-000 Manaus, AM, Brasil. \\ (krolow@inpa.gov.br) \\ 4. Departamento de Zoologia e Genética, IB, Universidade Federal de Pelotas (UFPel), Caixa Postal 354, 96010-900 Pelotas, RS, Brasil. \\ (rodrigorickes@yahoo.com.br) \\ 5. Departamento de Microbiologia e Parasitologia, IB, UFPel. (bretanha@ufpel.tche.br)
}

\begin{abstract}
Survival and reproduction of Synthesiomyia nudiseta (Diptera, Muscidae). The reproduction and survival of Synthesiomyia nudiseta (Wulp, 1883) were studied between 22 and $28^{\circ} \mathrm{C}, 70 \%$ R.H. and $12 \mathrm{~h}$ photoperiod. The raw data were analyzed based on age-class, sex life table, Weibull distribution and entropy curve $(H)$. The intrinsic rate of increase $\left(r_{m}\right)$, finite rate of increase $(\lambda)$, net reproduction rate $\left(\mathrm{R}_{0}\right)$ and mean generation time $(\mathrm{T})$ were $0.534,1.7,59.439$ and 7.65 respectively, suggesting fast population growth in these conditions. That is caused by the high fecundity that was confirmed for this species with mean number of eggs/female of 266 and mean preoviposition period of 10.25 days. The Weibull survival model showed that the survival was not constant throughout the adults life: the mortality rate was more pronounced after the fourth and fifth week because both sexes had $\mathrm{H}$ values intermediate to those theoretically expected $(0.0-0.5)$, showing that the survival curve is rectangular (with more impact of mortality on the fourth and fifth week from pupal emergence). The presented results can be considered as base for detailed studies on dynamics and population growth of this species in natural habitats.
\end{abstract}

KEYWORDS. Demographic analysis, survival analysis, entropy.

RESUMO. A reprodução e a sobrevivência de Synthesiomyia nudiseta (Wulp, 1883) foram estudadas entre 22 e $28^{\circ} \mathrm{C}$, UR de $70 \%$ e fotoperíodo de $12 \mathrm{~h}$. Os dados foram analisados através de classes de idade, tabelas de vida de fecundidade, análise de sobrevivência pela distribuição de Weibull e análise das curvas de sobrevivência através do conceito de entropia $(\mathrm{H})$. A taxa intrínseca de aumento natural $\left(\mathrm{r}_{\mathrm{m}}\right)$, taxa finita de aumento $(\lambda)$, taxa de reprodução líquida $\left(\mathrm{R}_{0}\right)$ e tempo médio de uma geração $(\mathrm{T})$ foram 0,$534 ; 1,7 ; 59,439$ e 7,65 respectivamente, sugerindo rápido crescimento populacional nestas condições. Isso foi causado pela alta fecundidade, confirmando o que ocorre para esta espécie, com média de 266 ovos/fêmea e período de pré-oviposição de 10,25 dias. O modelo de Weibull demonstrou que a sobrevivência não foi constante ao longo da vida de adultos, com mortalidade pronunciada depois da quarta e quinta semanas e valores de $\mathrm{H}$ intermediários aos valores teóricos esperados $(0,0-0,5)$ para os ambos sexos, demonstrando que a curva de sobrevivência é retangular (com maior impacto da mortalidade na quarta e quinta semanas após a emergência). Os resultados apresentados podem ser considerados como base para estudos pormenorizados da dinâmica e do crescimento populacional desta espécie em hábitats naturais.

PALAVRAS-CHAVE. Análise demográfica, análise de sobrevivência, entropia.

Reprodução e sobrevivência são parâmetros importantes na história de vida das espécies animais pois definem o sentido de crescimento de suas populações (CAREY, 1993), além de influenciar suas inter-relações conforme as mudanças nas taxas destes atributos por idade específica (BEGON et al., 1996). Entretanto, as alterações nas taxas reprodutivas e de mortalidade são de difícil visualização em hábitats naturais (CAREY, 2001), principalmente no caso de dípteros necrófagos que colonizam recursos efêmeros e irregulares (HANSKY, 1987; Moura, 2004).

As taxas demográficas para insetos são obtidas em laboratório a partir da manutenção de coortes (CAREY, 2001) que são amostras de uma população em que os indivíduos passaram por um mesmo evento significativo (normalmente o nascimento, o início da reprodução ou a emergência, no caso dos insetos adultos) (CAREY, 1993). A partir de coortes é possível a determinação de modelos de crescimento populacional, integrando a sobrevivência e a reprodução a partir de tabelas de vida e de fertilidade (BEGON et al., 1996).

Espécies sinantrópicas normalmente apresentam alta adaptabilidade a ambientes modificados pela ação antrópica, colonizando hábitats próximos a centros urbanos devido à alta capacidade reprodutiva e de propagação que apresentam, desde que o recurso preferencial seja abundante (MARTINEZ-SÁnCHEZ et al., 2000). A análise de sobrevivência pode determinar o tempo necessário para a colonização de novos ambientes onde a oviposição irá ocorrer (ROFF, 1977).

Synthesiomyia nudiseta (Wulp, 1883) (Diptera, Muscidae) é uma espécie cosmopolita com ampla distribuição na Região Neotropical (CARVALHo et al., 2005), apresentando altos valores de sinantropia em diversos centros urbanos do Brasil (Linhares, 1981; D'Almeida, 1992; Mendes \& Linhares, 1993; Carvalho et al., 2002; LeANDRo \& D'Almeida, 2005). Além de serem predadoras facultativas, as larvas desta espécie possuem hábitos alimentares necrófagos (BOHART \& GRESSIT, 1951; SkIDMORE, 1985) com possibilidade de uso como indicadoras do intervalo pós-morte na entomologia médico-criminal (FrEIRE, 1914; JIRÓN et al., 1983; LORD et al., 1992; OlIVEIRA-COSTA et al., 2001; KRÜGER et al., 2002). Os adultos podem ser considerados vetores de agentes etiológicos como poliovírus, Escherichia coli (Migula) e Shygella dysenteriae (Shiga) (GREENBERG, 1971). 
Este trabalho objetivou determinar as taxas reprodutivas de $S$. nudiseta a partir de tabelas de vida de fertilidade e modelar as curvas de sobrevivência de machos e fêmeas desta espécie.

\section{MATERIAL E MÉTODOS}

Manutenção de uma população estoque. Adultos de $S$. nudiseta foram capturados no campus da Universidade Federal de Pelotas (UFPel) na cidade de Capão do Leão, Rio Grande do Sul, Brasil (31 ${ }^{\circ} 45^{\prime} 48^{\prime}$ 'S, $\left.52^{\circ} 29^{\prime} 02^{\prime \prime} \mathrm{W}\right)$ e mantidos em câmara com temperatura variando entre 22 e $28^{\circ} \mathrm{C}$, umidade relativa do ar de $75 \%$ e fotofase de 12 horas para obtenção de ovos, larvas e pupas. Os adultos foram acondicionados em gaiolas com 30x30x30cm $(\mathrm{n}=4)$ e alimentados com leite em pó, açúcar refinado e farinha de peixe, numa proporção de 2:2:1 (KRÜGER et al., 2002), oferecendo-se água em pequenos frascos com espuma de poliestireno cobrindo a superfície do líquido. A ração e a água foram oferecidas ad libitum.

As larvas foram alimentadas com dieta composta de cinco partes de farinha de peixe, três de farinha de trigo e duas de serragem (KRÜGER et al., 2002) em funis de criação com até $2 \mathrm{~kg}$ de dieta adicionando água para umedecê-la. Em cada gaiola estoque foi colocado um pote com $250 \mathrm{~g}$ de dieta que diariamente foi transferido para o funil, havendo controle da densidade. A renovação da colônia foi a partir das posturas da mesma. As pupas foram incubadas a $26^{\circ} \mathrm{C} \pm 1^{\circ} \mathrm{C}$ em serragem úmida até a emergência dos adultos.

Delineamento experimental. Para o estudo da sobrevivência e da reprodução dos adultos, foram mantidas quatro gaiolas em laboratório, com adultos oriundos de 240 pupas escolhidas aleatoriamente (60 pupas para cada gaiola) da quinta geração, acondicionadas conforme descrito anteriormente. As gaiolas foram observadas diariamente, removendo-se os insetos mortos e as posturas.

O sexo foi determinado após a morte dos insetos e a razão sexual (RS) foi obtida da seguinte forma: $R S=\frac{F}{M+F}$ onde $\mathrm{F}$ é o número de fêmeas e $\mathrm{M}$ é o número de machos. Foi realizado teste de aderência de qui-quadrado para razão sexual em cada gaiola levandose em consideração a hipótese nula de que a razão sexual em populações desta espécie é de 0,5 (um macho para cada fêmea) com $\mathrm{p}<0,05$.

Análise de sobrevivência. Os dados de mortalidade diária foram submetidos à análise de sobrevivência usando a distribuição de frequiências de Weibull (CRAWLEY, 1993). A diferença na sobrevivência de machos e fêmeas foi testada considerando a condição de $\mathrm{p}<0,05$; caso contrário foi admitido que não houve diferença entre os sexos.

Análise da estimativa de entropia $(\mathrm{H})$. Os tipos de curvas de sobrevivência foram avaliados de acordo com o conceito de entropia $(\mathrm{H})$. Para o cálculo de $\mathrm{H}$, foram utilizados os seguintes parâmetros, calculados a partir dos dados de sobrevivência em tabelas de vida (CAREY, 1993): x, classe etária compreendida em intervalos de sete dias; $1_{x}$, a proporção de sobreviventes em relação ao intervalo $x$ de idade; $d_{x}=1_{x}-1_{x+1}$ fornece a proporção de indivíduos que morreram entre $\mathrm{x}$ e $\mathrm{x}+1 ; \mathrm{q}_{\mathrm{x}}=\mathrm{d}_{\mathrm{x}} / 1_{\mathrm{x}}$, a probabilidade de morte na idade $x$ e, $p_{x}=1-q_{x}$, a probabilidade de sobrevivência. A probabilidade média de sobrevivência foi calculada por $\mathrm{L}_{\mathrm{x}}=\mathrm{l}_{\mathrm{x}}-\left(\mathrm{d}_{\mathrm{x}} / 2\right)$; o número total de dias restantes aos sobreviventes até a idade máxima $\mathrm{w}, \mathrm{T}_{\mathrm{x}}$, foi estimado por $\sum_{y=x}^{w} L_{y}$ e a esperança de vida $\left(\mathrm{e}_{\mathrm{x}}\right.$ ) estimada por $\mathrm{T}_{\mathrm{x}} / 1_{\mathrm{x}}$. Os valores de entropia $(\mathrm{H})$ foram calculados seguindo-se a expressão $H=\frac{\sum_{x=0}^{w} e_{x} d_{x}}{e_{0}}$ onde $e_{x}$ representa a esperança de vida na idade $x ; d_{x}$ a proporção de indivíduos que morreram entre as idades $x$ e $\mathrm{x}+1 ; \mathrm{e}_{0}$ a esperança de vida na idade inicial e $\mathrm{w}$ a idade máxima alcançada pelo último indivíduo sobrevivente (CAREY, 1993).

Tabela de vida de fecundidade. Foi construída uma tabela de vida de fecundidade conforme CAREY (1993), onde (x) é a classe etária desde ovo até a última morte, medida em semanas; $\left(1_{x}\right)$ taxa de sobrevivência; $\left(m_{x}\right)$ é a taxa de ovos por fêmea por semana e que originarão fêmeas, conforme $m_{x}=($ ovos / fêmeas $) \times R S$.

A taxa reprodutiva líquida $\left(\mathrm{R}_{0}\right)$ foi obtida por $R_{0}=\sum l_{x} m_{x}$, onde $\mathrm{x}$ é a classe etária em um dado momento $i$. O tempo médio de geração (T) é dado por $T=\frac{\sum l_{x} m_{x} x}{\sum l_{x} m_{x}} \quad$ e a taxa intrínseca de aumento natural $\left(\mathrm{r}_{\mathrm{m}}\right)$ é dada por $r_{m}=\frac{\ln \left(R_{0}\right)}{T}$. A taxa finita de aumento $(\lambda)$ foi estimada a partir de $\lambda=\mathrm{e}^{\mathrm{r}_{\mathrm{m}}}$.

A $1_{x}$ e $x$ dos estágios imaturos foram obtidos da viabilidade total e período de desenvolvimento de ovo a adulto estimado por KRÜGER et al. (2002) para esta espécie nas mesmas condições de manutenção mencionadas anteriormente.

Espécimes-testemunho que emergiram da criação em laboratório foram depositados na Coleção de Entomologia "Pe. Jesus Santiago Moure", Departamento de Zoologia (DZUP), Universidade Federal do Paraná.

\section{RESULTADOS}

Não houve diferença na razão sexual (RS) nas quatro gaiolas (Tab. I). O período de pré-oviposição foi de 10,25 $\pm 1,48$ dias e média do número de ovos por fêmeas que ovipositaram de $266,34 \pm 57,75$ ovos

Até a quarta semana, cerca de $75 \%$ dos ovos foram ovipositados, aumentando para cerca de $91 \%$ até a quinta semana. Nas três últimas semanas de vida das fêmeas, menos de $1 \%$ dos ovos foram depositados na dieta (Tab. II). A taxa de ovos por fêmea que originaram fêmeas $\left(m_{x}\right)$ foi maior na terceira e quarta semanas, mantendo-se acima de 28 ovos por fêmea da segunda à sexta semana (Tab. II).

A partir dos dados sobre sobrevivência e reprodução (Tab. II) foi possível estimar a taxa reprodutiva líquida $\left(\mathrm{R}_{0}\right)$, o tempo médio de uma geração $(\mathrm{T})$, a taxa intrínseca de aumento natural $\left(\mathrm{r}_{\mathrm{m}}\right)$ e a taxa finita de aumento $(\lambda)$. Nas condições deste experimento, a coorte aumentou 59,4 vezes $\left(\mathrm{R}_{0}\right)$ a partir do seu tamanho inicial em 7,65 semanas (T), com acréscimo de 1,7 fêmeas por fêmea por semana $(\lambda)$. A partir dos valores de $R_{0}$ e $T$, a contribuição por individuo ao crescimento da coorte $\left(\mathrm{r}_{\mathrm{m}}\right)$ foi de 0,534 .

A longevidade não diferiu entre machos e fêmeas $\left(\chi^{2}=0,34 ; \mathrm{GL}=1 ; \mathrm{p}=0,56 ; \mathrm{n}=197\right)$ pela distribuição de Weibull com mesmo termo médio para a morte do modelo para ambos os sexos $(\mu=36,82)$ a partir da análise de sua distribuição. O modelo de sobrevivência dos adultos foi 
$l_{x}=e^{-36,82^{-2,29} x^{2,29}}$, onde $l_{x}$ é a proporção de sobreviventes em um dado período x (Fig. 1).

As curvas de sobrevivência para machos e fêmeas de S. nudiseta seguiram um padrão retangular, com uma tendência da mortalidade ocorrer em idades mais avançadas conforme a análise de entropia, sendo que a curva foi um pouco mais convexa para os machos $(H=0,327)$ do que para as fêmeas ( $H=0,342$ ) (Fig. 2, Tab. III).

$\mathrm{O}$ produto $\mathrm{e}_{\mathrm{x}} \mathrm{d}_{\mathrm{x}}$, que representa a média ponderada da $e_{x}$ em idades específicas foi maior entre os machos na quarta e quinta semanas e entre as fêmeas na primeira e quinta semanas (Tab. IV). Caso as mortes que ocorreram nestas semanas fossem evitadas, haveria a adição de cinco e seis dias na expectativa de vida inicial $\left(\mathrm{e}_{0}\right)$ de machos e fêmeas, respectivamente.

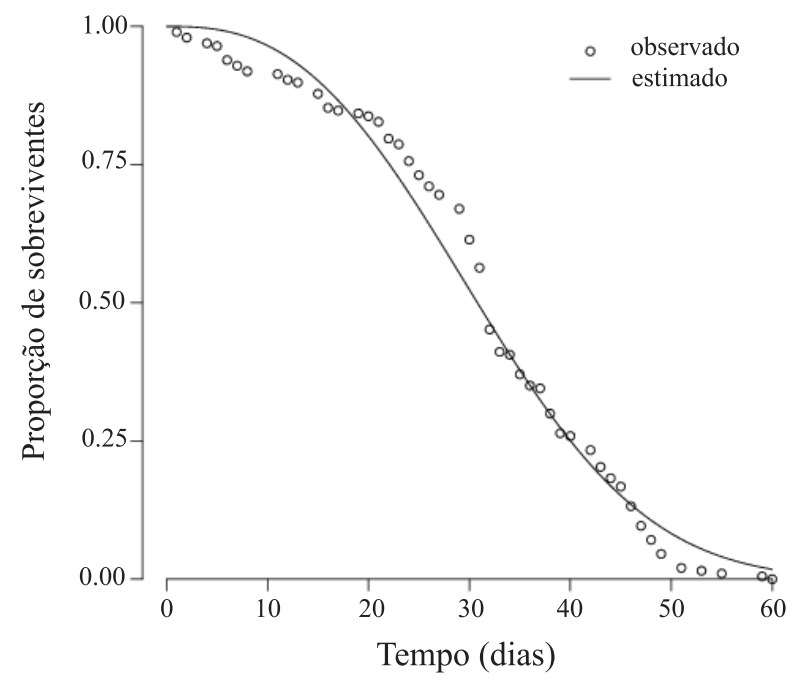

Fig. 1. Curva de sobrevivência para os adultos de Synthesiomyia nudiseta entre 22 e $28^{\circ} \mathrm{C}$, umidade relativa do ar acima de $75 \%$ e fotofase de 12 horas em condições de laboratório com a estimativa do modelo de distribuição de Weibull $\left(l_{x}=e^{-36,82^{-2,29} x^{2,29}}\right)$ $\left(x^{2}=0,34 ; \mathrm{GL}=1 ; \mathrm{p}=0,56 ; \mathrm{n}=197\right)$ ( $\mathrm{GL}$, graus de liberdade; 1 , proporção de sobreviventes por idade $\mathrm{x}$; $\mathrm{p}$, probabilidade; $\mathrm{n}$, númerơ de indivíduos; $x^{2}$, qui-quadrado).

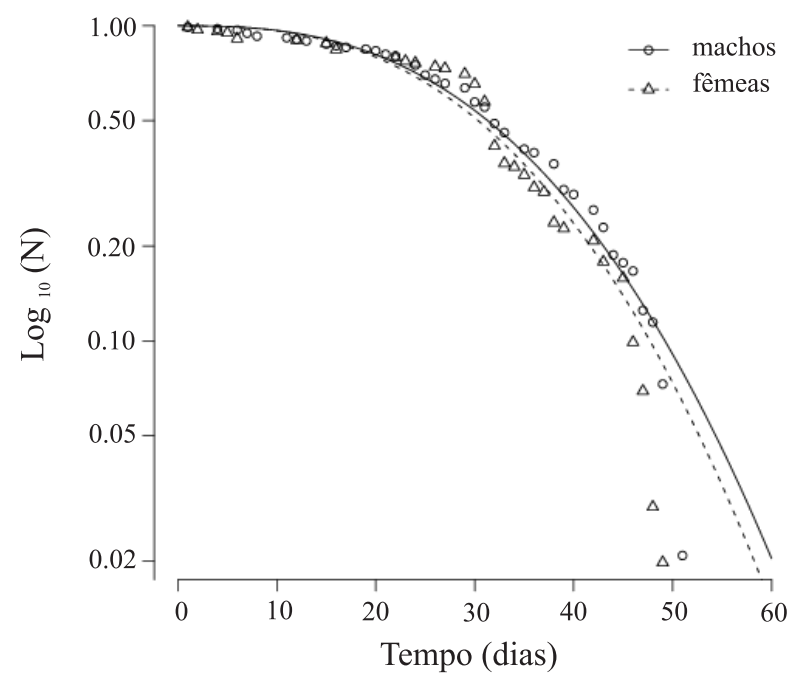

Fig. 2. Curva de sobrevivência de Synthesiomyia nudiseta em função do período de vida. Valores de entropia $(H)$ para machos $(0,327)$ e fêmeas $(0,342)$ entre 22 e $28^{\circ} \mathrm{C}$, umidade relativa do ar acima de $75 \%$ e fotofase de 12 horas em condições de laboratório ( $\mathrm{N}$, número de indivíduos vivos).
Tabela I. Número total de machos, fêmeas e ovos de Synthesiomyia nudiseta entre 22 e $28^{\circ} \mathrm{C}$, umidade relativa do ar acima de $75 \%$ e fotofase de 12 horas em condições de laboratório por colônia (ठ’, número de machos; + , número de fêmeas; $\Sigma$ a, somatória de adultos; $\Sigma$ o, somatória de ovos; RS, razão sexual; ns, não significativo pelo teste de qui-quadrado; $\chi^{2}$, teste de qui-quadrado com $\left.\mathrm{p}<0,05\right)$.

\begin{tabular}{lcccccc}
\hline Gaiolas & $\mathrm{O}^{\pi}$ & \& & $\Sigma \mathrm{a}$ & $\Sigma \mathrm{o}$ & $\mathrm{RS}$ & $\chi^{2}$ \\
\hline $\mathrm{A}$ & 28 & 25 & 53 & 4.655 & 0,47 & $\mathrm{~ns}$ \\
$\mathrm{~B}$ & 20 & 17 & 33 & 4.972 & 0,46 & $\mathrm{~ns}$ \\
$\mathrm{C}$ & 20 & 28 & 48 & 6.458 & 0,58 & $\mathrm{~ns}$ \\
D & 28 & 31 & 59 & 7.691 & 0,53 & $\mathrm{~ns}$ \\
$\Sigma$ & 96 & 101 & 197 & 23.776 & 0,51 & \\
\hline
\end{tabular}

Tabela II. Tabela de fecundidade das colônias de Synthesiomyia nudiseta em função do intervalo etário entre 22 e $28^{\circ} \mathrm{C}$, umidade relativa do ar acima de $75 \%$ e fotofase de 12 horas em condições de laboratório ( $F_{x}$, número de fêmeas; $1_{x}$, sobrevivência; $m_{x}$, taxa de ovos por fêmeas e que originaram fêmeas; $\mathrm{NTO}_{\mathrm{x}}$, número total de ovos; X, semana). * Foi utilizado o valor de 0,49 para sobrevivência inicial na quinta semana conforme KRÜGER et al. (2002).

\begin{tabular}{rrrcc}
\hline $\mathrm{X}$ & $\mathrm{F}_{\mathrm{x}}$ & $\mathrm{NTO}_{\mathrm{x}}$ & $\mathrm{m}_{\mathrm{x}}$ & $\mathrm{l}_{\mathrm{x}}^{*}$ \\
\hline 5 & 101 & 0 & 0 & 0,49 \\
6 & 92 & 5042 & 28,24 & 0,45 \\
7 & 91 & 6288 & 35,61 & 0,44 \\
8 & 85 & 6556 & 39,75 & 0,41 \\
9 & 74 & 3878 & 27,01 & 0,36 \\
10 & 34 & 1869 & 28,33 & 0,17 \\
11 & 21 & 75 & 1,84 & 0,10 \\
12 & 3 & 54 & 9,28 & 0,02 \\
13 & 2 & 14 & 3,61 & 0,01 \\
\hline
\end{tabular}

Tabela III. Parâmetros utilizados para o cálculo da entropia $(\mathrm{H})$ de machos $\left({ }^{\top}\right)$ e fêmeas () de Synthesiomyia nudiseta entre 22 e $28^{\circ} \mathrm{C}$, umidade relativa do ar acima de $75 \%$ e fotofase de 12 horas em condições de laboratório por colônia (e, esperança de vida na idade inicial; $\mathrm{W}$, idade máxima em dias alcançada pelo último indivíduo sobrevivente; $\Sigma \mathrm{e}_{\mathrm{x}} \mathrm{d}_{\mathrm{x}}$, somatória das médias ponderadas da esperança de vida em todas as idades).

\begin{tabular}{cll}
\hline & \multicolumn{1}{c}{$0^{\top}$} & \multicolumn{1}{c}{+} \\
\hline $\mathrm{e}_{\mathrm{o}}$ & 31,630 & 30,312 \\
$\mathrm{~W}$ & 55 & 60 \\
$\Sigma \mathrm{e}_{\mathrm{x}} \mathrm{d}_{\mathrm{x}}$ & 10,341 & 10,356 \\
\hline
\end{tabular}

Tabela IV. Valores do produto $\mathrm{e}_{\mathrm{x}} \mathrm{d}_{\mathrm{x}}$ sobre intervalos de sete dias (X) para machos $\left(0^{7}\right)$ e fêmeas (†) de Synthesiomyia nudiseta entre 22 e $28^{\circ} \mathrm{C}$, umidade relativa do ar acima de $75 \%$ e fotofase de 12 horas em condições de laboratório por colônia.

\begin{tabular}{ccc}
\hline $\mathrm{X}$ & $\sigma^{\top}$ & q \\
\hline 1 & 0,991 & 2,823 \\
2 & 1,889 & 0,271 \\
3 & 1,314 & 1,221 \\
4 & 2,767 & 1,599 \\
5 & 2,417 & 3,670 \\
6 & 1,553 & 1,151 \\
7 & 1,063 & 0,930 \\
8 & 0,385 & 0,082 \\
9 & 0,991 & 0,070 \\
\hline
\end{tabular}




\section{DISCUSSÃO}

Foram reconhecidos três períodos na história de vida dos adultos de S. nudiseta: pré-oviposição, oviposição e pós-oviposição ou período de senescência. O período de pré-oviposição, que envolve o tempo necessário para a maturação dos ovócitos e as primeiras atividades de cópula, refletiu os valores encontrados para outras espécies necrófagas de muscídeos, mantidos nas mesmas condições de criação dos adultos desta espécie (RABINOVICH, 1970; RIBEIRO et al., 2000; KRÜGER et al., 2004). A variação nesta estimativa ocorre, além das condições de manutenção da colônia, por causa da dieta e número de casais utilizados (Tirone et al., 1996; D’Almeida et al., 1997). A manutenção de casais em grupos tem o inconveniente da perda de controle do período de pré-oviposição por indivíduo, mas em contrapartida aumenta a probabilidade de cópulas devido ao maior número de indivíduos presentes.

A alta capacidade reprodutiva de $S$. nudiseta está de acordo com o que foi avaliado anteriormente para esta espécie (RABINOVICH, 1970; D’Almeida et al., 1997). Este período de oviposição ocorreu com maior intensidade entre a segunda e sexta semana de vida dos adultos, com taxas mais elevadas $\left(\mathrm{m}_{\mathrm{x}}\right)$ até a quinta semana, conforme o verificado para outras espécies de Muscidae (RIBEIRO et al., 2000; KRÜGER et al., 2004).

O terceiro período de vida dos adultos de $S$. nudiseta é caracterizado pela brusca diminuição nas taxas reprodutivas, seguido de alta mortalidade. A partir da quarta semana, ainda durante o período de oviposição, o impacto da mortalidade na expectativa de vida de machos e fêmeas começa a ser observado através do produto $e_{x} d_{x}$. Esta tendência da mortalidade ocorrer no final do período de oviposição, seguindo durante o período de pós-oviposição para ambos os sexos acentuou a convexidade das curvas de sobrevivência (Fig. 2). Este padrão, analisado através do conceito de entropia $(\mathrm{H})$ ocorre em outras moscas necrófagas, em análises quantitativas (Moura \& BonATTO, 1999; FernANDEs et al., 2003; KRÜGER \& ERTHAL, 2006) e qualitativas da distribuição de mortes (D’AlmeIDA \& AlmeIDA, 1996; RIBEIRO et al., 2000; KRÜGER et al., 2004).

A análise de entropia permite predizer que, caso houvesse a redução de $1 \%$ na mortalidade de $S$. nudiseta, haveria a adição de 0,327 e 0,342 dias na expectativa de vida de machos e fêmeas, respectivamente, como preconizado por outros autores (DEMETRIUS, 1978, 1979; GOLDMAN \& LORD, 1986; MOURA \& BONATTO, 1999; KRÜGER \& Erthal, 2006). Não existem testes estatísticos que permitam verificar a robustez das afirmações a respeito dos valores observados em relação aos valores teóricos de entropia. Em relação aos valores de $\mathrm{H}$ obtidos para $S$. nudiseta neste trabalho, as afirmações de que a curva de sobrevivência de machos e fêmeas desta espécie é do tipo retangular, conforme teorizado por DEMETRIUS (1978), são corroboradas pelo termo $\alpha$ da distribuição de Weibull. Quando $\alpha>1$, a taxa de mortalidade aumenta com o passar do tempo.

A história de vida dos adultos desta espécie segue o padrão verificado para outras espécies de dípteros necrófagos, com alta oviposição em duas ou três semanas, seguida de alta mortalidade. A relação entre alta reprodução e baixa mortalidade nas primeiras semanas de vida dos adultos permite uma alta adaptabilidade e forte tendência de crescimento populacional $\left(\mathrm{r}_{\mathrm{m}}, \mathrm{R}_{0} \mathrm{e} \lambda\right)$ nas condições de criação.

Agradecimentos. À CAPES, pela bolsa concedida a RFK; ao PIBIC/CNPq, pelas bolsa concedidas a TKK e JLPD.

\section{REFERÊNCIAS BIBLIOGRÁFICAS}

Begon, M.; Mortimer, M. \& Thompson, D. J. 1996. Population ecology. An unified study of animals and plants. Cambridge, Blackwell. 247p.

Bohart, G. E. \& Gressit, J. L. 1951. Filth-inhabiting flies of Guam. Honolulu, Bernice P. Bishop Museum (Bulletin 204). $152 \mathrm{p}$.

CAREY, J. R. 1993. Applied demography for biologists: with special emphasis on insects. New York, Oxford University. 206 p.

2001. Insect biodemography. Annual Review of Entomology 46:79-110.

Carvalho, C. J. B. De; Moura, M. O. \& Ribeiro, P. B. 2002. Chave para adultos de dípteros (Muscidae, Fanniidae, Anthomyiidae) associados ao ambiente humano no Brasil. Revista Brasileira de Entomologia 46(1):107-114.

Carvalho, C. J. B. de; Couri, M. S.; Pont, A. C.; Pamplona, D. \& Lopes, S. M. 2005. A catalogue of the Muscidae (Diptera) of Neotropical region. Zootaxa 860:1-282.

Crawley, M. J. 1993. GLIM for ecologists. Oxford, Blackwell. $761 \mathrm{p}$.

D’Almeida, J. M. 1992. Calyptrate Diptera (Muscidae and Anthomyidae) of the State of Rio de Janeiro - I. Synanthropy. Memórias do Instituto Oswaldo Cruz 87(3):381-386.

D’Almeida, J. M. \& AlmeidA, J. R. DE. 1996. Longevidade e curva de sobrevivência de oito espécies de dípteros caliptrados (Calliphoridae, Muscidae e Sarcophagidae), em condições de laboratório. Revista Brasileira de Biologia 56(3):497-505.

D’Almeida, J. M.; Piana, M. L. G. \& Selem, C. T. 1997 Comportamento reprodutivo de Synthesiomyia nudiseta van der Wulp (Diptera: Muscidae) sob condições de laboratório. Memórias do Instituto Oswaldo Cruz 92(4):563-564.

Demetrius, L. 1978. Adaptative value, entropy and survivorship curve. Nature 275:213-214.

1979. Relations between demographic parameters Demography 16(2):329-338.

Fernandes, F. M.; Lapola, D. M.; Neregato, R.; Carvalho, M. C. \& Von Zuben, C. J. 2003. Curva de sobrevivência e estimativa de entropia em Lucilia cuprina (Diptera, Calliphoridae). Iheringia, Série Zoologia, 93(3):319-324.

Freire, O. 1914. Algumas notas para o estudo da fauna cadavérica da Bahia. Gazeta Médica da Bahia 46(3):110-125.

Goldman, N. \& Lord, J. W. 1986. A new look at entropy and the life table. Demography 23(2):275-282.

Greenberg, B. 1971. Flies and disease. Ecology, classification and biotic associations. New Jersey, Princeton University. v.1. $586 \mathrm{p}$.

HANsky, I. 1987. Carrion fly community dynamics: patchiness, seasonality and coexistence. Ecological Entomology 12(3):257-266.

Jirón, L. F.; Vargas, L. G. \& Vargas-Alvarado, E. 1983. Four muscoid flies (Sarcophagidae and Muscidae) associated with human cadavers in Costa Rica. Brenesia 21:3-5.

KRÜGer, R. F. \& ERthal, S. G. 2006. Estimativa de entropia da Muscina stabulans (Fallén) (Diptera, Muscidae) em condições artificiais. Revista Brasileira de Entomologia 50(2):275-279.

Krüger, R. F.; Ribeiro, P. B.; Carvalho, C. J. B. DE \& Costa, P. R. P. DA. 2002. Desenvolvimento de Synthesiomyia nudiseta (Diptera, Muscidae) em laboratório. Iheringia, Série Zoologia, 92(4):25-30.

Krüger, R. F.; Ribeiro, P. B.; Carvalho, C. J. B. De; Lambrecht, F. M. \& Nunes, A. M. 2004. Longevidade e oviposição de Ophyra albuquerquei Lopes (Diptera, Muscidae) em condições de laboratório. Iheringia, Série Zoologia, 94(2):211-216. 
Leandro, M. J. F. \& D’Almeida, J. M. 2005. Levantamento de Calliphoridae, Fanniidae, Muscidae e Sarcophagidae em um fragmento de mata na Ilha do Governador, Rio de Janeiro, Brasil. Iheringia, Série Zoologia, 95(4):377-381.

Linhares, A. X. 1981. Synanthropy of Muscidae, Fanniidae and Anthomyiidae (Diptera) in the city of Campinas, São Paulo, Brazil. Revista Brasileira de Entomologia 25(4):231-243.

Lord, W. D.; Adkins, T. R. \& Catts, E. P. 1992. The use of Synthesiomyia nudiseta (Van Der Wulp) (Diptera, Muscidae) and Calliphora vicina (Robineau-Desvoidy) (Diptera, Calliphoridae) to estimate the time of death of a body buried under a house. Journal of Agriculture Entomology 9(4):227-235.

Martínez-Sánchez, A.; Rojo, S. \& Marcos-García, M. A. 2000. Annual and spatial activity of dung flies and carrion in a Mediterranean holm-oak pasture ecosystem. Medical and Veterinary Entomology 14(1):56-63.

Mendes, J. \& Linhares, A. X. 1993. Atratividade por iscas, sazonalidade e desenvolvimento ovariano em várias espécies de Muscidae (Diptera). Revista Brasileira de Entomologia 37(2):289-297.

Moura, M. O. 2004. Variação espacial como mecanismo promotor da coexistência em comunidades de insetos necrófagos. Revista Brasileira de Zoologia 21(3):409-419.
Moura, M. O. \& Bonatto, S. R. 1999. Análise de sobrevivência e estimativa de entropia para Sarconesia chlorogaster (Wiedemann) (Diptera, Calliphoridae). Revista Brasileira de Zoologia 16(Supl.1):221-226.

Oliveira-Costa, J.; Mello-Patiú, C. A. \& Lopes, S. M. 2001. Dípteros muscóides associados com cadáveres humanos no local da morte, no Estado do Rio de Janeiro, Brasil. Boletim do Museu Nacional, Série Zoologia, 464:1-6.

Rabinovich, J. 1970. Vital statistics of Synthesiomyia nudiseta (Diptera: Muscidae). Annals of Entomological Society of America 63(3):749-752.

Ribeiro, P. B.; Carvalho, C. J. B. de; Chernaki, A. M. \& Costa, P. R. P. DA. 2000. Longevidade, oviposição e viabilidade pupal de Ophyra aenescens Wiedemann, 1830 (Diptera, Muscidae, Azeliinae), em condições de laboratório. Revista Brasileira de Agrociência 6(3):264-268.

RoFF, D. 1977. Dispersal in dipterans: its costs and consequences. Journal of Animal Ecology 46(2):443-456.

Skidmore, P. 1985. The biology of the Muscidae of the world. Dordrecht, Dordrecht Kunk. 550p.

Tirone, G.; Parise, P. P.; Avancini, R. M. P. 1996. Ovarian development in Muscina stabulans (Diptera, Muscidae) on differents diets. Revista Brasileira de Entomologia 40(1): 105-106.

Recebido em agosto de 2006. Aceito em setembro de 2007. ISSN 0073-4721

Artigo disponível em: www.scielo.br/isz 